\title{
Ellagic acid-Fe@BSA nanoparticles for preferential payload delivery and chemodynamic therapy in A549 cells
}

\author{
Sandeep Suresh Menon, Sivaramakrishnan Venkatabalasubramanian* (D) \\ Department of Genetic Engineering, SRM Institute of Science and Technology, Chennai-603205, Tamil Nadu, India.
}

\begin{tabular}{l}
\hline ARTICLE INFO \\
\hline Article history: \\
Received on: May 18, 2021 \\
Accepted on: July 24, 2021 \\
Available online: November 10, 2021 \\
\hline
\end{tabular}

Key words:

Lung cancer, nanoparticles, ellagic acid, A549, Fenton reaction

\begin{abstract}
Recent advances in nanomedicine hail chemodynamic therapy (CDT), based on the Fe (II)-mediated Fenton reaction, as a forthcoming, potentially revolutionary, and tumor mediation strategy. Various experiments have proven the therapeutic effects of CDT in vivo as well as in diverse tumor cell lines, but most processes lack significant targeting among other hurdles, viz., the optimization of physical parameters (i.e., aggregation, organic carriers, conjugates etc.). Briefly, $\mathrm{Fe}$ (III) in the form of $\mathrm{FeCl}_{3}$ was conjugated with bovine serum albumin nanoparticles (NP) in order to derive a novel in vitro nano-therapeutic system against A549 cells by a chemical synthesis approach. A naturally occurring anti-tumor agent ellagic acid was conjugated to the NPs to improve their therapeutic effect and test for the anticipated increase in bioavailability and cytotoxicity of the drug. The NP system was sufficiently characterized using dynamic light scattering, UV-vis spectroscopy, and Fourier transform infrared spectroscopy analysis and cell viability was examined using an (3-(4,5-dimethylthiazol2-yl)-2,5-diphenyltetrazolium bromide) assay. The results demonstrate the NP system to be within range of proper nano-delivery mechanism and cytotoxicity examinations reveal the novel drug and CDT combination to be greatly effective in reducing tumor cell proliferation, holding great promise in being extrapolated to in vivo model systems for further analysis.
\end{abstract}

\section{INTRODUCTION}

Lung cancer is observed to be among the most prevalent type of cancers in India, second to only to breast and oral cancers. This is, unsurprisingly, mostly due to the socio-cultural decay in terms of poor lifestyle choices, and in part due to the growing plethora of associated risk factors, making it uniquely evident in India. Mortality rates differ based on the aggressiveness of the cancer while the prognostic viability of treatments depend greatly on the type of lung tissue affected. A benign combination of both is seen in non-small cell lung cancers (NSCLCs) which progress slowly but account for a large portion of lung cancer fatalities. Taking this into account, the A549 cell line was employed as the model system for conducting the in vitro analyses of this project.

\footnotetext{
*Corresponding Author

Sivaramakrishnan Venkatabalasubramanian, Department of Genetic Engineering, SRM Institute of Science and Technology, Chennai-603205, Tamil Nadu, India.E-mail: sivaramv@srmist.edu.in
}

Previous studies in this realm have been largely focused on the neo-synthesis of receptor-specific drug molecules to stimulate premature apoptosis in the affected cells or to synthesize the likeness of metabolic inhibitors to block off essential cellular pathways. However, in the turn of the last decade, cytosolic administration of organic micro and nano vessels conjugated with apoptotic or inhibitory drug molecules have been driven into the spotlight - the advent of nanoparticle systems came with a promise for a more efficient drug delivery system. Recent developments have thoroughly proven the efficiency of conjugated nanoparticles (NP) in effectively limiting the proliferation of high Insp8-expressing $\mathrm{HCT} 116$ cells using $\mathrm{Cu}_{2} \mathrm{O}$ NPs [1], and again using Fe@BSA NPs [2]. Interestingly, the use of Fe (III) as the inorganic basis for building a NP system provided useful insight into the rather underappreciated subcellular Fenton reaction [3]. This involved the $\mathrm{Fe}$ (II) mediated catalysis of the conversion of endogenous hydrogen peroxide $\left(\mathrm{H}_{2} \mathrm{O}_{2}\right)$ to cytotoxic hydroxyl free radicals $(\bullet \mathrm{OH})$. Taking advantage of this property, we aimed to synthesize and study the basic effects of a Fe-based nano-system on the effective subcellular conversion of $\mathrm{H}_{2} \mathrm{O}_{2}$ to $\cdot \mathrm{OH}$ in A549 cells, better known as chemodynamic therapy (CDT). 
Menon and Venkatabalasubramanian: Ellagic acid—Fe@BSA nanoparticles for preferential payload delivery and chemodynamic therapy ih45 A549 cells 2021;9(06):144-148

The $\cdot \mathrm{OH}$ is a more oxidizing reactive oxygen species than $\mathrm{H}_{2} \mathrm{O}_{2}$, evident from standard electrode potentials $(E)$ of 2.80 and $1.78 \mathrm{~V}$, respectively, thus resulting in devastating oxidative damage at the tumor site [2]. However, the extent of the damage is largely based on sufficient endogenous concentrations of $\mathrm{H}_{2} \mathrm{O}_{2}$ and unchallenged, swift endogenous conversion of $\mathrm{Fe}$ (III) to $\mathrm{Fe}$ (II) to catalyze the forward reaction. The uncertainty in this aspect proved worrisome for the overall success of the NP system.

Instead of directly promoting CDT efficacy, this problem was tackled by conjugating a novel drug viz. ellagic acid (EA) to the nano-mechanism in order to further promote tumor suppression. EA - a condensed dimer of gallic acid - is an innately occurring polyphenol antioxidant in various nuts and berries which has been later found to substantially limit the proliferation of certain tumors. Studies performed using HCT116 and Caco-2 cell lines have shown EA-induced growth inhibition to be a result of the upregulation p53 and p21 genes and reduced production of insulin like growth factor-II causing apoptosis due to a reduction in adenosine triphosphate production [4]. In A549 cell lines, the inhibition of the PI3K/Akt signaling pathway was suspected to be an additional antitumor mechanism [4]. Its monomeric equivalent, gallic acid, shows minor apoptotic properties in similar concentrations compared to EA.

Another important aspect of the NP system is the use of bovine serum albumin (BSA) as the carrier of choice. BSA has a homologous 3D structure to HSA, and therefore is suspected to improve on the stealth characteristics of the nano-system in vivo. Albumin nano-carriers also bind negatively or positively charged inorganic compounds non-covalently, allowing significant amounts of drug molecules to be incorporated onto or encapsulated within them and vastly improving its release profile both in vitro and in vivo [5]. BSA derivates are also smaller compared to other liposomal nano-carriers, and hence, generally have a more controlled payload delivery property.

Therefore, the NP-mediated strategy developed against A549 cells in this project will provide valuable information on the individual aspects of this novel targeting system which can later be extrapolated to reducing proliferation of other forms cancerous lung tissue.

\section{MATERIALS AND METHODS}

\subsection{Materials}

Crystalline $\mathrm{FeCl}_{3}$ hexahydrate, BSA and EA (96\%) used were of analytical reagent grade and purchased from SRL Chemicals (Mumbai, India). Adenocarcinomic human alveolar basal epithelial cells (A549) used in this study were purchased for research purpose from National Centre for Cell Sciences (Pune, India) and maintained at SRM Institute of Science and Technology as per standard guidelines mentioned by American type culture collection in Dulbecco's Modified Eagle Medium (GIBCO, Schwalbach, Germany), supplemented with $10 \%$ fetal bovine serum (GIBCO, Schwalbach, Germany), 100 units/ml Penicillin, $100 \mathrm{mg} / \mathrm{ml}$ Streptomycin, pH 7.4 in $25 \mathrm{~cm}^{2}$, tissue culture flasks (Tarson's, Kolkata, India) at $37^{\circ} \mathrm{C}$ under $5 \% \mathrm{CO}_{2}$ and $95 \%$ air.

\subsection{Synthesis of EA-Fe@BSA NPs}

The methodology for generation of NPs was adapted from the overnight chemical synthesis protocol [2]. Deionized water was used to dissolve $66.6 \mathrm{mg}$ of BSA using a magnetic stirrer. Once sufficiently solvate was generated, $108 \mu \mathrm{l}$ of $0.1 \mathrm{~g} / \mathrm{ml}$ aqueous $\mathrm{FeCl}_{3}$ was added, and the mixture was allowed to stand at room temperature and pressure under stirring conditions for 30 minutes. An alcoholic (ethanolic) solution of $1,002 \mu 1$ of EA $(10 \mathrm{mg} / \mathrm{ml})$ was then introduced into the still stirring mixture in a drop-wise manner. The solution was left overnight on the magnetic stirrer until a dark blue color was observed, confirming the formation of NPs.

\subsection{Characterization}

UV-vis spectrometry (ThermoFisher, GENESYS 150) was used to approximate the relative composition of the NP system, in terms of concentrations gauged from the absorbance values, between 200 and $1,100 \mathrm{~nm}$. dynamic light scattering (DLS) analysis (Horiba Scientific, SZ-100) using a dispersion medium of viscosity 0.894 $\mathrm{mPa} \cdot \mathrm{s}$, count rate of $3,131 \mathrm{kCPS}$ and scattering angle of $90^{\circ}$ at $25^{\circ} \mathrm{C}$ was used to ascertain the size, dispersity, and hydrodynamic diameter of the NP system with and without coating EA. The molecular profile of the NP system was determined using the Fourier transform infrared spectroscopy (FTIR) (Shimadzu, IRTracer-100) spectrum at a $2 \mathrm{~cm}^{-1}$ resolution run over 25 scans for analysis.

\subsection{Biocompatibility}

The cytotoxicity of the NPs toward A549 cells was analyzed using an (3-(4,5-dimethylthiazol-2-yl)-2,5-diphenyltetrazolium bromide) (MTT) assay. A 96-well plate was inoculated with 100 $\mu \mathrm{l}$ of $105 / \mathrm{ml}$ cells into 12 wells. After incubating for 24 hours, $150 \mu \mathrm{l}$ aliquot triplicates of three distinct EA-Fe@BSA NP concentrations - 50, 250 and $500 \mu \mathrm{M}$ - contained in Dulbecco's modified Eagle medium medium were added. The triplicate serving as the control was inoculated with media only. After a further 24 hours, the supernatant in the wells were aspirated and MTT solution was added before incubating the plate for the next 4 hours. Finally, the supernatant was once again removed and the wells were rinsed well with phosphate buffered saline before adding dimethyl sulfoxide and measuring the absorbance using a microplate reader (ThermoFisher, Multiskan FC). The plate was maintained at $37^{\circ} \mathrm{C}$ in a $5 \% \mathrm{CO}_{2}$ incubator throughout.

\section{RESULTS AND DISCUSSION}

\subsection{Synthesis}

During the initial addition of $\mathrm{FeCl}_{3}$ to the BSA, a straw-colored mixture was observed. Upon addition of the ethanolic solution of EA, the color of the solution was seen to turn dark blue, affirming the formation of NPs [2]. After the overnight incubation period, the color was seen to darken further.

\subsection{UV-Vis Spectrometry}

Absorbance values at 425 and $875 \mathrm{~nm}$ for the NP system, 20 hours after the addition of EA, were seen to be 3.508 and 3.016, respectively (Fig. 1 and Table 1). This revealed no 
UV-vis Spectrum [NP]

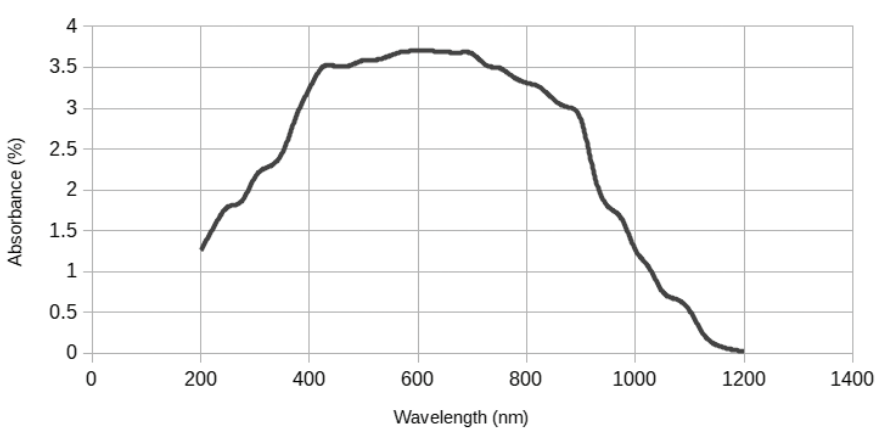

Figure 1: UV-vis spectrum (EA-Fe@BSA NP).

Table 1: UV-vis results.

\begin{tabular}{lcc} 
Sample & Wavelength $(\mathbf{n m})$ & Absorbance \\
\hline EA-Fe@BSA NP & 425 & 3.508 \\
& 609 & 3.721 \\
& 875 & 3.016 \\
$\mathrm{FeCl}_{3}$ & 334 & 3.278 \\
& 425 & 0.027 \\
$\mathrm{EA}$ & 875 & 0.003 \\
& 254 & 3.411 \\
& 283 & 3.926 \\
& 425 & 1.145 \\
& 875 & 0.200 \\
\hline
\end{tabular}

UV-vis Spectrum [Iron (III) Chloride]

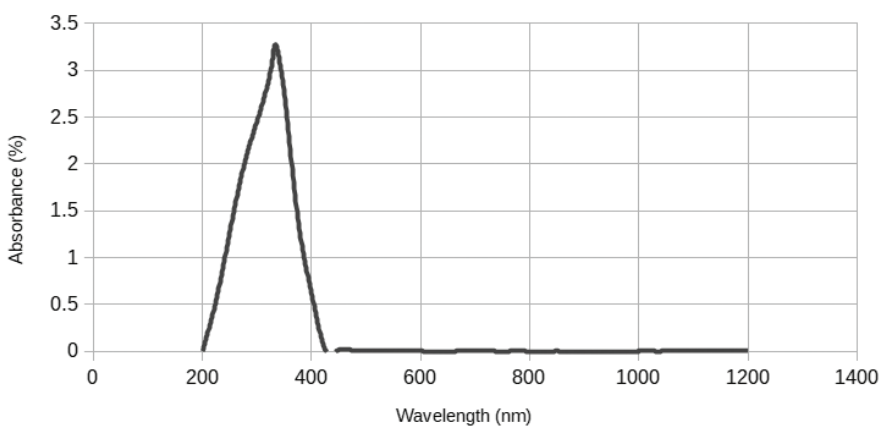

Figure 2: UV-vis spectrum $\left(\mathrm{FeCl}_{3}\right.$, Gray).

significant aggregation in the synthesized NP after the latest addition of EA [6], and the highest peak obtained at $609 \mathrm{~nm}$ may be attributed to the characteristic $d$ - $d$ electronic transition consistent with the formation of an EA-Fe complex. $\mathrm{FeCl}_{3}$ showed high absorbance peaks between 300 and $400 \mathrm{~nm}$ (Fig. 2 and Table 1), proving no external contaminations but possibly the presence of excess, unconverted Fe(III) [7]. Two absorbance peaks of 3.411 and 3.926 were observed for EA at $254 \mathrm{~nm}$ and $283 \mathrm{~nm}$, respectively, in Figure 3 which is within the expected, standard range for a neutral $\mathrm{pH}$-range solution [8]. However, an absorbance of 1.145 at $425 \mathrm{~nm}$ was also observed, which is suspected to be residual EA having been dissociated from the EA-Fe complex.

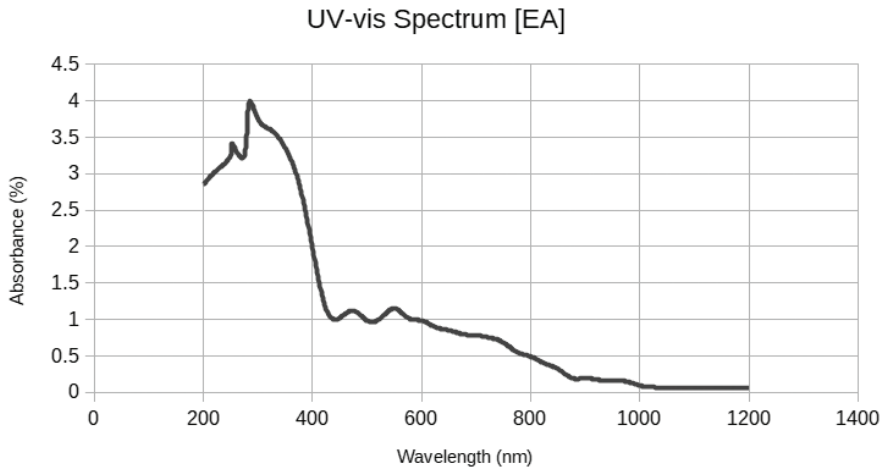

Figure 3: UV-vis spectrum (EA, gray).

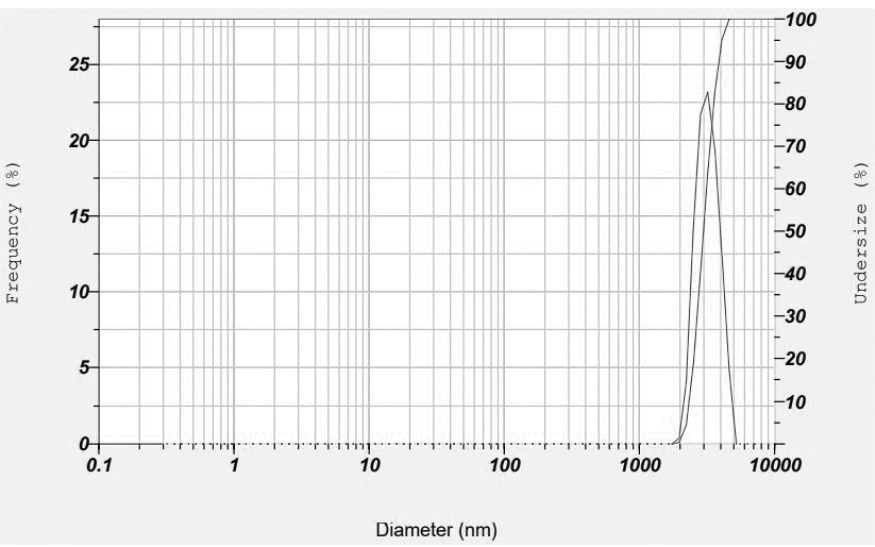

Figure 4: DLS result (Fe@BSA).

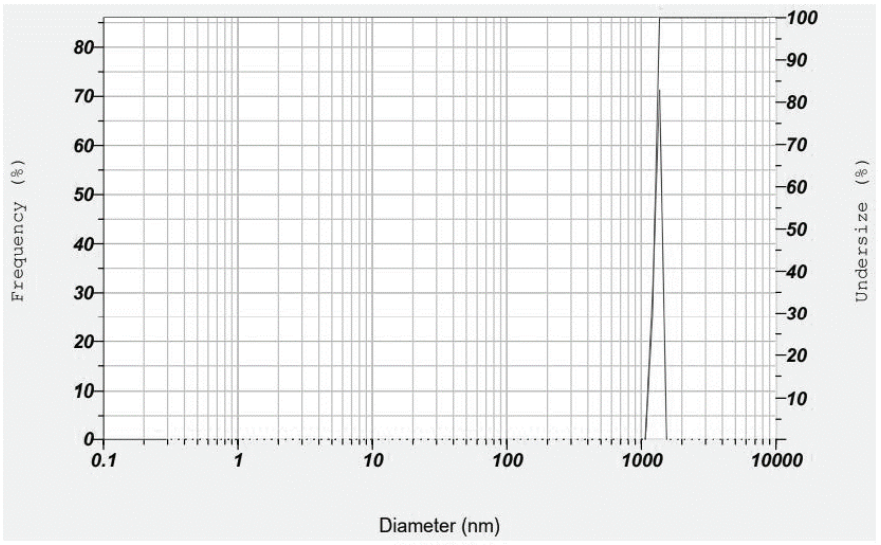

Figure 5: DLS result (EA-Fe@BSA NP).

\subsection{DLS}

The NP system showed a polydisperse distribution with a mean hydrodynamic diameter of $881.9 \mathrm{~nm}$ (Fig. 5). However, due to their polydisperse nature, it is difficult to conclude, accurately, the diameter of a monodisperse particle. This is yet well within range of an effective in vitro delivery system [2]. It is also far removed from the 1,715.2 $\mathrm{nm}$ monodisperse form of the non-conjugated carrier (Fig. 4 and Table 2), further cementing the nanoscale size of the NP system. 
Menon and Venkatabalasubramanian: Ellagic acid—Fe@BSA nanoparticles for preferential payload delivery and chemodynamic therapy ih47 A549 cells 2021;9(06):144-148

Table 2: DLS results.

\begin{tabular}{lcc} 
Sample & Z-average $(\mathbf{n m})$ & PI \\
EA-Fe@BSA NP & 881.9 & 2.360 \\
$\mathrm{Fe} @$ BSA & $1,715.2$ & 2.950 \\
\hline
\end{tabular}

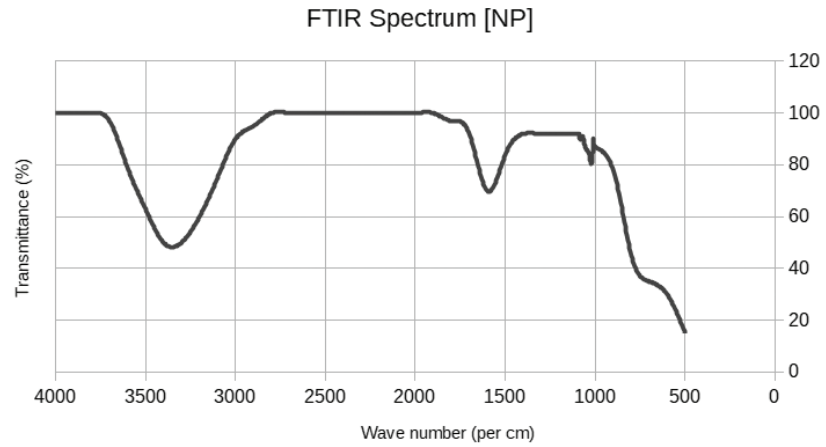

Figure 6: FTIR spectrum (EA-Fe@BSA NP).

Table 3: FTIR results.

\begin{tabular}{cc} 
Wave number $\left(\mathbf{c m}^{-1}\right)$ & Transmittance (\%) \\
$1,044.47$ & 85 \\
$1,084.98$ & 90 \\
$1,639.52$ & 70 \\
$3,300.00$ & 50 \\
\hline
\end{tabular}

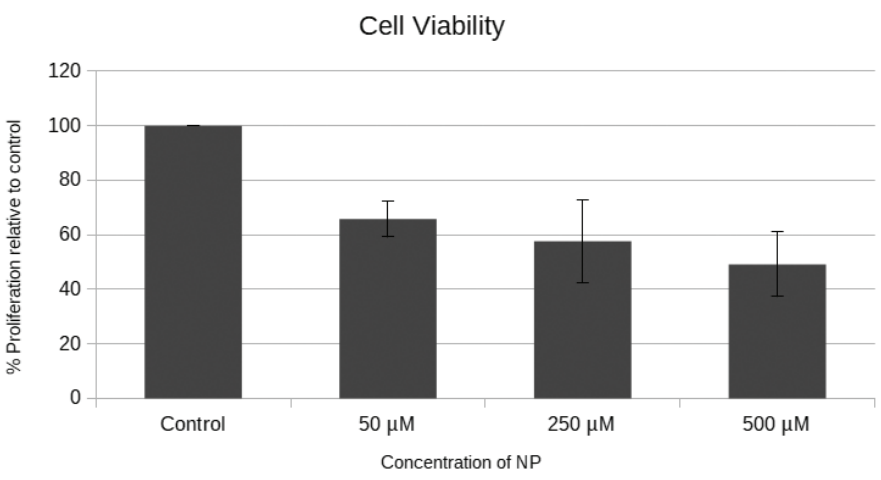

Figure 7: A549 proliferation relative to control at $0,50,250$ and $500 \mu \mathrm{M}$ EAFe@BSA NP.

\subsection{FTIR}

The bands at $1,640 \mathrm{~cm}^{-1}$ show evidence of amide band vibrations, hence confirming the presence of BSA. Furthermore, the NP system exhibited distinct bands at 1,085 and 1,044 $\mathrm{cm}^{-1}$ (Fig. 6), demonstrating a spectrum relatively close to that of pure EA $\left(1,196\right.$ and $\left.1,039 \mathrm{~cm}^{-1}\right)$ derived from the characteristic $\mathrm{C}-\mathrm{O}$ bond stretching. The fingerprint region of the spectrum additionally revealed the $\mathrm{OH}$ stretching band of EA in the range of 3,000-3,500 $\mathrm{cm}^{-1}$ (Table 3) thus, proving fortunate chelation of the catechol moieties of EA to Fe (II) and Fe (III) ions [2].

\subsection{MTT assay}

As evident from the MTT assay (Fig. 7), the viability of A549 cells was seen to trend downward with increasing concentrations of EAFe@BSA NPs. The wide range between individual NP dosages administered was chosen to make the correlation more obvious. Although the administration variability per concentration on average is high at higher doses, there is a clear and steady decline in cell proliferation up to $51 \%$ at $500 \mu \mathrm{M}$ indicating that the efficacy of the EA-CDT combination in suppressing NSCLC progression is strong and promising. Therefore, the novel NP system exhibits excellent biocompatibility and specific cytotoxicity against A549 cells, thus promising much hope for further in vivo testing.

\section{CONCLUSION}

In a nutshell, a novel, nanoscale EA-Fe@BSA nano-therapeutic system showing good biocompatibility was synthesized using a simple chemical synthesis approach and effectively characterized to provide finer compositional detail. The combination of the natural polyphenol and homologue albumin showed good stability and minimal aggregation for extended periods at room temperature in a NP form, while the addition of Fe (III) was, within reason, suspected to emphasize specific cytotoxicity of the system by enhanced CDT.

The ease of synthesis of this NP system, coupled with the ability to replace the inorganic component with more magnetic residues, gives way to new horizons in personalized nanomedicine. Ferrimagnetic NPs and SPIONs have already been established in the realm of targeted drug delivery but comprise of a complicated generation and purification process. The novel NP system developed in this project aims not only to prove its effectiveness against specific cancerous tissue, but also to make its facets readily available for rapid testing and applications. This system exploits both endogenous processes and naturally extracted therapeutics to guide cooperation among them using new generation nanotechnology, providing an encouraging paradigm for the treatment of NSCLC.

\section{AUTHOR CONTRIBUTIONS}

SSM and VSRK contributed to the conception, drafting, and editing of the manuscript. All authors confirm the integrity of the manuscript.

\section{FUNDING}

There is no funding to report.

\section{CONFLICT OF INTEREST}

The authors report no financial or any other conflicts of interest in this work.

\section{ETHICAL APPROVALS}

This study does not involve experiments on animals or human subjects. 


\section{REFERENCES}

1. Gnanavela V, Palanichamy V, Roopana SM. Biosynthesis and characterization of copper oxide nanoparticles and its anticancer activity on human colon cancer cell lines (HCT-116). J Photochem Photobiol B 2017;171:133-8.

2. Tian Q, An L, Tian Q, Lin J, Yang S. Ellagic acid-Fe@BSA nanoparticles for endogenous $\mathrm{H} 2 \mathrm{~S}$ accelerated $\mathrm{Fe}(\mathrm{III}) / \mathrm{Fe}$ (II) conversion and photothermal synergistically enhanced chemodynamic therapy. Theranostics 2020;10(9):4101-15.

3. Kalidasan V, Liu XL, Herng TS, Yang Y, Ding J. Bovine serum albumin-conjugated ferrimagnetic iron oxide nanoparticles to enhance the biocompatibility and magnetic Hyperthermia performance. Nanomicro Lett 2015; 8:80-93.

4. Liu Q, Liang X, Niu C, Wang X. Ellagic acid promotes A549 cell apoptosis via regulating the phosphoinositide 3-kinase/protein kinase B pathway. Exp Ther Med 2018; 16(1):347-52.

5. Bronze-Uhle ES, Costa BC, Ximenes VF, Lisboa-Filho PN. Synthetic nanoparticles of bovine serum albumin with entrapped salicylic acid. Nanotechnol Sci Appl 2016;10:11-21.
6. Ríos -Corripio MA, García-Pérez BE, Jaramillo-Flores ME, Gayou VL, Rojas-López M. UV-Visible intensity ratio (aggregates/single particles) as a measure to obtain stability of gold nanoparticles conjugated with protein A. J Nanoparticle Res 2013;15(5) :1624-30.

7. Abderrazak H, Dachraoui M, Lendl B. A novel flow injection procedure for determination of phosphate in industrial raw phosphoric acid. Analyst 2000;125(6):1211-3.

8. Simić AZ, Verbić TŽ, Sentić MN. Study of ellagic acid electrooxidation mechanism. Monatsh Chem 2013;144:121-8.

\section{How to cite this article:}

Menon SS, Venkatabalasubramanian S. Ellagic acid-Fe@,

BSA nanoparticles for preferential payload delivery and chemodynamic therapy in A549 cells. J Appl Biol Biotech 2021; 9(06):144-148. 